\title{
Covering the integers by arithmetic sequences
}

\author{
by
}

\author{
ZHI WeI Sun (Nanjing)
}

1. Introduction. Let $\mathbb{R}$ be the field of real numbers and $\mathbb{R}^{+}$the set of positive reals. For $\alpha \in \mathbb{R}$ and $\beta \in \mathbb{R}^{+}$we call

$$
\alpha+\beta \mathbb{Z}=\{\ldots, \alpha-2 \beta, \alpha-\beta, \alpha, \alpha+\beta, \alpha+2 \beta, \ldots\}
$$

an arithmetic sequence with common difference $\beta$. In the case $\alpha \in \mathbb{Z}$ and $\beta \in \mathbb{Z}^{+}, \alpha+\beta \mathbb{Z}$ is just the residue class $\alpha \bmod \beta$ with modulus $\beta$.

Let $m$ be a positive integer. A finite system

$$
\mathcal{A}=\left\{\alpha_{s}+\beta_{s} \mathbb{Z}\right\}_{s=1}^{k} \quad\left(\alpha_{1}, \ldots, \alpha_{k} \in \mathbb{R} \text { and } \beta_{1}, \ldots, \beta_{k} \in \mathbb{R}^{+}\right)
$$

of arithmetic sequences is said to be an (exact) $m$-cover of $\mathbb{Z}$ if it covers each integer at least (resp., exactly) $m$ times. Instead of "1-cover" and "exact 1-cover" we use the terms "cover" and "exact cover" respectively.

Since they were introduced by P. Erdös ([5]) in the early 1930's, covers of $\mathbb{Z}$ by (finitely many) residue classes have been studied seriously and many nice applications have been found. (Cf. sections A19, B21, E23, F13 and F14 of R. K. Guy [9].) For problems and results in this area we refer the reader to surveys of Erdős [7, 8], Š. Porubský [13] and Š. Znám [21]. Recently further progress was made by various authors.

If a finite system

$$
A=\left\{a_{s}+n_{s} \mathbb{Z}\right\}_{s=1}^{k} \quad\left(a_{1}, \ldots, a_{k} \in \mathbb{Z} \text { and } n_{1}, \ldots, n_{k} \in \mathbb{Z}^{+}\right)
$$

of residue classes forms an $m$-cover of $\mathbb{Z}$, then $\sum_{s=1}^{k} 1 / n_{s} \geq m$, and the equality holds if and only if $(2)$ is an exact $m$-cover of $\mathbb{Z}$. This becomes apparent if we calculate

$\sum_{s=1}^{k}\left|\left\{0 \leq x<N: x \equiv a_{s}\left(\bmod n_{s}\right)\right\}\right|=\sum_{x=0}^{N-1}\left|\left\{1 \leq s \leq k: x \equiv a_{s}\left(\bmod n_{s}\right)\right\}\right|$

where $N$ is the least common multiple of $n_{1}, \ldots, n_{k}$.

Research supported by the National Nature Science Foundation of P.R. China. 
In this paper we investigate properties of $m$-covers of $\mathbb{Z}$ in the form (1). In the next section we shall give three equivalent conditions for (1) to be an $m$-cover of $\mathbb{Z}$. One is that (1) covers $W$ consecutive integers at least $m$ times where

$$
W=\left|\left\{\left\{\sum_{s \in I} \frac{1}{\beta_{s}}\right\}: I \subseteq\{1, \ldots, k\}\right\}\right|
$$

( $[x]$ and $\{x\}$ stand for the integral and fractional parts of a real $x$ respectively throughout the paper), the other two are finite systems of equalities (not inequalities) involving roots of unity. Our tools used to deduce them include Vandermonde determinants, Stirling numbers, a little analysis and linear algebra.

In Sections 3 and 4 we will derive a number of results including the following ones:

(I) Let (1) be an $m$-cover of $\mathbb{Z}$ and $J \subseteq\{1, \ldots, k\}$. Then

$$
\left\{\sum_{s \in I} \frac{1}{\beta_{s}}\right\}=\left\{\sum_{s \in J} \frac{1}{\beta_{s}}\right\} \quad \text { for some } I \subseteq\{1, \ldots, k\} \text { with } I \neq J
$$

provided $\sum_{s=1}^{k} 1 / \beta_{s}=m$ (e.g. (1) is an exact $m$-cover of $\mathbb{Z}$ with $\alpha_{s} \in \mathbb{Z}$ and $\beta_{s} \in \mathbb{Z}^{+}$for $\left.s=1, \ldots, k\right)$ we have $\sum_{s \in I} 1 / \beta_{s}=\sum_{s \in J} 1 / \beta_{s}$ for some $I \subseteq$ $\{1, \ldots, k\}$ with $I \neq J$ if $\emptyset \neq J \subset\{1, \ldots, k\}$, when $\sum_{s \in I} 1 / \beta_{s}=\sum_{s \in J} 1 / \beta_{s}$ for no $I \subseteq\{1, \ldots, k\}$ with $I \neq J$ there are at least $m$ nonzero integers of the form $\sum_{s \in I} 1 / \beta_{s}-\sum_{s \in J} 1 / \beta_{s}$ where $I \subseteq\{1, \ldots, k\}$.

(II) Let $k \geq l \geq 0$ be integers. Then $2^{k-l}(l+1)$ is the smallest $n \in \mathbb{Z}^{+}$ such that any system of $k$ arithmetic sequences with at least $l$ equal common differences covers an arithmetic sequence at least $m$ times if it covers $n$ consecutive terms in the sequence at least $m$ times.

The last section contains some unsolved problems related to possible extensions.

2. Characterizations of $m$-covers. Let us provide several technical lemmas the first of which serves as the starting point of our new approach.

Lemma 1. Let $m \in \mathbb{Z}^{+}$and $x \in \mathbb{R}$. Then (1) covers $x$ at least $m$ times if and only if

$$
\prod_{s=1}^{k}\left(1-r^{1 / \beta_{s}} e^{2 \pi i\left(\alpha_{s}-x\right) / \beta_{s}}\right)=o\left((1-r)^{m-1}\right) \quad(r \rightarrow 1) .
$$

Proof. Set $I=\left\{1 \leq s \leq k: x \in \alpha_{s}+\beta_{s} \mathbb{Z}\right\}$ and $I^{\prime}=\{1, \ldots, k\} \backslash I$. 
Clearly,

$$
\begin{aligned}
\lim _{r \rightarrow 1} \frac{\prod_{s=1}^{k}\left(1-r^{1 / \beta_{s}} e^{2 \pi i\left(\alpha_{s}-x\right) / \beta_{s}}\right)}{(1-r)^{|I|}} & \\
= & \lim _{r \rightarrow 1} \prod_{s \in I^{\prime}}\left(1-r^{1 / \beta_{s}} e^{2 \pi i\left(\alpha_{s}-x\right) / \beta_{s}}\right) \cdot \lim _{r \rightarrow 1} \prod_{s \in I} \frac{1-r^{1 / \beta_{s}}}{1-r} \\
& =\left.\prod_{s \in I^{\prime}}\left(1-e^{2 \pi i\left(\alpha_{s}-x\right) / \beta_{s}}\right) \cdot \prod_{s \in I} \frac{d}{d r}\left(r^{1 / \beta_{s}}\right)\right|_{r=1} \\
& =\prod_{s \in I^{\prime}}\left(1-e^{2 \pi i\left(\alpha_{s}-x\right) / \beta_{s}}\right) \cdot \prod_{s \in I} \beta_{s}^{-1} \neq 0
\end{aligned}
$$

and hence

$$
\begin{aligned}
\lim _{r \rightarrow 1} \frac{\prod_{s=1}^{k}\left(1-r^{1 / \beta_{s}} e^{2 \pi i\left(\alpha_{s}-x\right) / \beta_{s}}\right)}{(1-r)^{m-1}} \\
\quad=\lim _{r \rightarrow 1} \frac{\prod_{s=1}^{k}\left(1-r^{1 / \beta_{s}} e^{2 \pi i\left(\alpha_{s}-x\right) / \beta_{s}}\right)}{(1-r)^{|I|}}(1-r)^{|I|-m+1} \\
= \begin{cases}0 & \text { if }|I|>m-1, \\
\infty & \text { if }|I|<m-1 .\end{cases}
\end{aligned}
$$

Now it is apparent that $|I| \geq m$ if and only if (3) holds. We are done.

Lemma 2. Let $\theta_{1}, \ldots, \theta_{n}$ be real numbers with distinct fractional parts. For any $\varepsilon>0$ there exists a $\delta>0$ such that if

$$
\left|\sum_{t=1}^{n} e^{2 \pi i s \theta_{t}} x_{t}\right|<\delta
$$

for every $s=1, \ldots, n$ then $\left|x_{t}\right|<\varepsilon$ for all $t=1, \ldots, n$.

Proof. Let $A$ be the matrix $\left(e^{2 \pi i s \theta_{t}}\right)_{\substack{1 \leq s \leq n \\ 1 \leq t \leq n}}$. Then

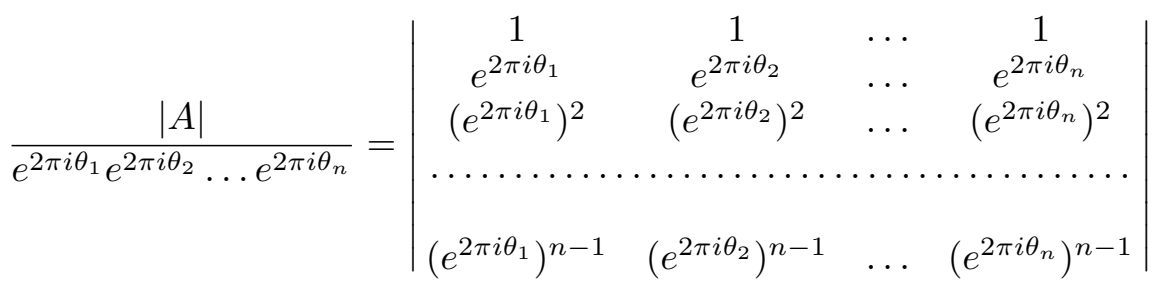

is a determinant of Vandermonde's type. As $|A| \neq 0$ the inverse matrix of $A$ exists; we denote it by $B=\left(b_{s t}\right)_{\substack{1 \leq s \leq n \\ 1 \leq t \leq n}}$.

Let $b=\max \left\{\left|b_{s t}\right|: s, t=1, \ldots, n\right\}>0$ and $\delta=\varepsilon /(b n)$. Let $x_{1}, \ldots, x_{n}$ 
be any complex numbers, and set

$$
y_{s}=\sum_{t=1}^{n} e^{2 \pi i s \theta_{t}} x_{t} \quad \text { for } s=1, \ldots, n .
$$

Let

$$
\vec{x}=\left(\begin{array}{c}
x_{1} \\
\vdots \\
x_{n}
\end{array}\right) \quad \text { and } \quad \vec{y}=\left(\begin{array}{c}
y_{1} \\
\vdots \\
y_{n}
\end{array}\right)
$$

Then $\vec{x}=B A \vec{x}=B \vec{y}$. If $\left|y_{s}\right|<\delta$ for every $s=1, \ldots, n$, then

$$
\left|x_{s}\right|=\left|\sum_{t=1}^{n} b_{s t} y_{t}\right| \leq \sum_{t=1}^{n} b\left|y_{t}\right|<b n \delta=\varepsilon \quad \text { for all } s=1, \ldots, n .
$$

This concludes the proof.

Lemma 3. Let $m \in \mathbb{Z}^{+}$. Then

$$
\sum_{n=0}^{m-1} a_{n} t^{n-m+1}=o(1) \quad(t \rightarrow 0)
$$

if and only if $a_{0}=\ldots=a_{m-1}=0$.

Pr o of. The "if" direction is trivial. When $a_{0}, \ldots, a_{m-1}$ are not all zero, for the least $k$ such that $a_{k} \neq 0$ we have

$$
\sum_{n=0}^{m-1} a_{n}\left(x^{-1}\right)^{n-m+1}=\sum_{n=k}^{m-1} a_{n} x^{m-1-n} \sim a_{k} x^{m-1-k} \quad(x \rightarrow \infty),
$$

which contradicts (4). This ends the proof.

Lemma 4. Let $n \geq m>0$ be integers and $a_{1}, \ldots, a_{n}$ distinct numbers. Then the system

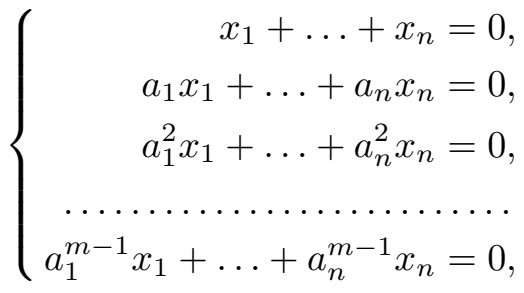

is equivalent to

$$
\left\{\begin{array}{c}
a_{11} x_{1}+\ldots+a_{1 n} x_{n}=0 \\
a_{21} x_{1}+\ldots+a_{2 n} x_{n}=0 \\
\ldots \ldots \ldots \ldots \ldots \ldots \ldots+\ldots \\
a_{m 1} x_{1}+\ldots+a_{m n} x_{n}=0
\end{array}\right.
$$


where

$$
a_{s t}=\prod_{\substack{i=1 \\ i \neq s}}^{m} \frac{a_{i}-a_{t}}{a_{i}-a_{s}} \quad \text { for } s=1, \ldots, m \text { and } t=1, \ldots, n \text {. }
$$

Pro of. Rewrite (5) in the form

$$
\left\{\begin{aligned}
x_{1}+\ldots+x_{m} & =-\sum_{m<t \leq n} x_{t}, \\
a_{1} x_{1}+\ldots+a_{m} x_{m} & =-\sum_{m<t \leq n} a_{t} x_{t}, \\
a_{1}^{2} x_{1}+\ldots+a_{m}^{2} x_{m} & =-\sum_{m<t \leq n} a_{t}^{2} x_{t}, \\
\ldots \ldots \ldots \ldots \ldots \ldots \ldots \ldots & \ldots \ldots \ldots \ldots \ldots \\
{ }^{m-1} x_{1}+\ldots+a_{m}^{m-1} x_{m} & =-\sum_{m<t \leq n} a_{t}^{m-1} x_{t} .
\end{aligned}\right.
$$

By Cramer's rule, this says that

$$
\begin{aligned}
& x_{s}=\left|\begin{array}{ccccccc}
1 & \ldots & 1 & -\sum_{m<t \leq n} x_{t} & 1 & \ldots & 1 \\
a_{1} & \ldots & a_{s-1} & -\sum_{m<t \leq n} a_{t} x_{t} & a_{s+1} & \ldots & a_{m} \\
a_{1}^{2} & \ldots & a_{s-1}^{2} & -\sum_{m<t \leq n} a_{t}^{2} x_{t} & a_{s+1}^{2} & \ldots & a_{m}^{2} \\
\ldots \ldots \ldots & \ldots & \ldots \ldots \ldots \ldots \ldots \ldots \ldots \ldots \ldots \ldots & \ldots \ldots \ldots \ldots \ldots & \ldots \ldots \\
a_{1}^{m-1} & \ldots & a_{s-1}^{m-1} & -\sum_{m<t \leq n} a_{t}^{m-1} x_{t} & a_{s+1}^{m-1} & \ldots & a_{m}^{m-1}
\end{array}\right| \\
& \times\left|\begin{array}{ccc}
1 & \ldots & 1 \\
a_{1} & \ldots & a_{m} \\
a_{1}^{2} & \ldots & a_{m}^{2} \\
\ldots \ldots & \ldots & \ldots \\
a_{1}^{m-1} & \ldots & a_{m}^{m-1}
\end{array}\right|^{-1} \\
& =-\sum_{m<t \leq n} x_{t}\left|\begin{array}{ccccccc}
1 & \ldots & 1 & 1 & 1 & \ldots & 1 \\
a_{1} & \ldots & a_{s-1} & a_{t} & a_{s+1} & \ldots & a_{m} \\
a_{1}^{2} & \ldots & a_{s-1}^{2} & a_{t}^{2} & a_{s+1}^{2} & \ldots & a_{m}^{2} \\
\ldots \ldots & \ldots & \ldots \ldots \ldots \ldots \ldots \ldots \ldots \ldots \ldots & \ldots \ldots \ldots \ldots \\
a_{1}^{m-1} & \ldots & a_{s-1}^{m-1} & a_{t}^{m-1} & a_{s+1}^{m-1} & \ldots & a_{m}^{m-1}
\end{array}\right| \\
& \times\left|\begin{array}{ccc}
1 & \ldots & 1 \\
a_{1} & \ldots & a_{m} \\
a_{1}^{2} & \ldots & a_{m}^{2} \\
\ldots \ldots \ldots \ldots & \ldots \ldots \ldots \\
a_{1}^{m-1} & \ldots & a_{m}^{m-1}
\end{array}\right|^{-1}
\end{aligned}
$$




$$
\begin{aligned}
=-\sum_{m<t \leq n} x_{t} \frac{\prod_{1 \leq i<s}\left(a_{t}-a_{i}\right) \cdot \prod_{s<i \leq m}\left(a_{i}-a_{t}\right) \cdot \prod_{\substack{1 \leq i<j \leq m \\
i, j \neq s}}\left(a_{s}-a_{i}\right) \cdot \prod_{\substack{1 \leq s \\
s<i \leq m}}\left(a_{i}-a_{s}\right) \cdot \prod_{\substack{1 \leq i<j \leq m \\
i, j \neq s}}\left(a_{j}-a_{i}\right)}{\text { (Vandermonde) }} \\
=-\sum_{m<t \leq n} a_{s t} x_{t}
\end{aligned}
$$

for every $s=1, \ldots, m$, i.e.

$$
\sum_{t=1}^{m} \delta_{s t} x_{t}+\sum_{m<t \leq n} a_{s t} x_{t}=0 \quad \text { for } s=1, \ldots, m
$$

where $\delta_{s t}$ is the Kronecker delta. Since $a_{s t}=\delta_{s t}$ for $s, t=1, \ldots, m$, we have finished the proof.

Now we are ready to present

Theorem 1. Let $\mathcal{A}=\left\{\alpha_{s}+\beta_{s} \mathbb{Z}\right\}_{s=1}^{k}$, where $\alpha_{1}, \ldots, \alpha_{k} \in \mathbb{R}$ and $\beta_{1}, \ldots, \beta_{k} \in \mathbb{R}^{+}$. Let $m \in \mathbb{Z}^{+}$and

$$
S=\left\{0 \leq \theta<1:\left\{\sum_{s \in I} \frac{1}{\beta_{s}}\right\}=\theta \text { for some } I \subseteq\{1, \ldots, k\}\right\} .
$$

Let

$$
V(\theta)=\left\{\sum_{s \in I} \frac{1}{\beta_{s}}: I \subseteq\{1, \ldots, k\} \text { and } \sum_{s \in I} \frac{1}{\beta_{s}}-\theta \in \mathbb{Z}\right\}
$$

and $U(\theta)$ be a set of $m$ distinct numbers comparable with $V(\theta)$ (i.e. $|U(\theta)|$ $=m$, and either $U(\theta) \subseteq V(\theta)$ or $U(\theta) \supseteq V(\theta))$. Then the following statements are equivalent:

(a) $\mathcal{A}$ is an $m$-cover of $\mathbb{Z}$.

(b) $\mathcal{A}$ covers $|S|$ consecutive integers at least $m$ times.

(c) For each $\theta \in S$,

$$
\sum_{\substack{I \subseteq\{1, \ldots, k\} \\
\left\{\Sigma_{s \in I} 1 / \beta_{s}\right\}=\theta}}(-1)^{|I|}\left(\begin{array}{c}
{\left[\sum_{s \in I} 1 / \beta_{s}\right]} \\
n
\end{array}\right) e^{2 \pi i \Sigma_{s \in I} \alpha_{s} / \beta_{s}}=0
$$

holds for every $n=0,1, \ldots, m-1$. (As usual $\left(\begin{array}{l}x \\ n\end{array}\right)$ denotes $\frac{x(x-1) \ldots(x-n+1)}{1 \cdot 2 \cdot \ldots \cdot(n-1) n}$.)

(d) For any $\theta \in S$,

$$
\sum_{v \in V(\theta)} a_{u v} f(v)=0 \quad \text { for all } u \in U(\theta),
$$


where

$$
a_{u v}=\prod_{\substack{x \in U(\theta) \\ x \neq u}} \frac{x-v}{x-u} \quad \text { and } \quad f(v)=\sum_{\substack{I \subseteq\{1, \ldots, k\} \\ \Sigma_{s \in I} 1 / \beta_{s}=v}}(-1)^{|I|} e^{2 \pi i \Sigma_{s \in I} \alpha_{s} / \beta_{s}} .
$$

Proof. $(\mathrm{a}) \Rightarrow(\mathrm{b})$. This is obvious.

(b) $\Rightarrow(\mathrm{c})$. Suppose that each of $x+1, \ldots, x+|S|$ is covered by $\mathcal{A}$ at least $m$ times, where $x$ is an integer. By Lemma 1 for every $n=1, \ldots,|S|$ we have

$$
\begin{aligned}
0= & \lim _{r \rightarrow 1} \frac{\prod_{s=1}^{k}\left(1-r^{1 / \beta_{s}} e^{2 \pi i\left(\alpha_{s}-x-n\right) / \beta_{s}}\right)}{(1-r)^{m-1}} \\
= & \lim _{r \rightarrow 1}\left((1-r)^{1-m}\right. \\
& \left.\times \sum_{I \subseteq\{1, \ldots, k\}}(-1)^{|I|} r^{\Sigma_{s \in I} 1 / \beta_{s}} e^{2 \pi i \Sigma_{s \in I}\left(\alpha_{s}-x\right) / \beta_{s}} e^{-2 \pi i n \Sigma_{s \in I} 1 / \beta_{s}}\right) \\
= & \lim _{r \rightarrow 1} \sum_{\theta \in S} F(r, \theta) e^{-2 \pi i n \theta},
\end{aligned}
$$

where

$$
F(r, \theta)=\sum_{\substack{I \subseteq\{1, \ldots, k\} \\\left\{\Sigma_{s \in I} 1 / \beta_{s}\right\}=\theta}}(-1)^{|I|} r^{\Sigma_{s \in I} 1 / \beta_{s}} e^{2 \pi i \Sigma_{s \in I} \alpha_{s} / \beta_{s}} e^{-2 \pi i x \theta} /(1-r)^{m-1} .
$$

Let $\varepsilon$ be an arbitrary positive number. By Lemma 2 there is an $\eta>0$ such that if

$$
\left|\sum_{\theta \in S} e^{-2 \pi i n \theta} x_{\theta}\right|<\eta
$$

for every $n=1, \ldots,|S|$ then $\left|x_{\theta}\right|<\varepsilon$ for all $\theta \in S$. Since

$$
\sum_{\theta \in S} F(r, \theta) e^{-2 \pi i n \theta}=o(1) \quad(r \rightarrow 1) \quad \text { for } n=1, \ldots,|S|,
$$

there exists a $\delta>0$ such that whenever $|r-1|<\delta$,

$$
\left|\sum_{\theta \in S} F(r, \theta) e^{-2 \pi i n \theta}\right|<\eta \quad \text { for all } n=1, \ldots,|S|
$$

and hence by the above $|F(r, \theta)|<\varepsilon$ for each $\theta \in S$. This shows that $\lim _{r \rightarrow 1} F(r, \theta)=0$ for every $\theta \in S$. 
For any $\theta \in S$ we have

$$
\begin{aligned}
& 0=\lim _{r \rightarrow 1} \sum_{I \subseteq\{1, \ldots, k\}}(-1)^{|I|} r^{\Sigma_{s \in I} 1 / \beta_{s}} e^{2 \pi i \Sigma_{s \in I} \alpha_{s} / \beta_{s}} /(1-r)^{m-1} \\
& \left\{\Sigma_{s \in I} 1 / \beta_{s}\right\}=\theta \\
& =\lim _{t \rightarrow 0} \sum_{\substack{I \subseteq\{1, \ldots, k\} \\
\left\{\Sigma_{s \in I} 1 / \beta_{s}\right\}=\theta}}(-1)^{|I|}(1-t)^{\left[\Sigma_{s \in I} 1 / \beta_{s}\right]+\theta} t^{1-m} e^{2 \pi i \Sigma_{s \in I} \alpha_{s} / \beta_{s}} \\
& =\lim _{t \rightarrow 0} \sum_{\substack{I \subseteq\{1, \ldots, k\} \\
\left\{\Sigma_{s \in I} 1 / \beta_{s}\right\}=\theta}}(-1)^{|I|} \sum_{n=0}^{\left[\Sigma_{s \in I} 1 / \beta_{s}\right]}\left(\begin{array}{c}
{\left[\sum_{s \in I} 1 / \beta_{s}\right]} \\
n
\end{array}\right)(-t)^{n} t^{1-m} e^{2 \pi i \Sigma_{s \in I} \alpha_{s} / \beta_{s}} \\
& =\lim _{t \rightarrow 0} \sum_{\substack{I \subseteq\{1, \ldots, k\} \\
\left\{\Sigma_{s \in I} 1 / \beta_{s}\right\}=\theta}}\left((-1)^{|I|} e^{2 \pi i \Sigma_{s \in I} \alpha_{s} / \beta_{s}}\right. \\
& \left.\times \sum_{\substack{n=0 \\
n \leq\left[\Sigma_{s \in I} 1 / \beta_{s}\right]}}^{m-1}\left(\begin{array}{c}
\left.\sum_{s \in I} 1 / \beta_{s}\right] \\
n
\end{array}\right)(-1)^{n} t^{n-m+1}\right) \\
& =\lim _{t \rightarrow 0} \sum_{n=0}^{m-1}(-1)^{n}\left(\sum_{\substack{I \subseteq\{1, \ldots, k\} \\
\left\{\Sigma_{s \in I} 1 / \beta_{s}\right\}=\theta}}(-1)^{|I|}\left(\begin{array}{c}
{\left[\sum_{s \in I} 1 / \beta_{s}\right]} \\
n
\end{array}\right) e^{2 \pi i \Sigma_{s \in I} \alpha_{s} / \beta_{s}}\right) t^{n-m+1} .
\end{aligned}
$$

In view of Lemma $3,(7)$ holds for every $n=0,1, \ldots, m-1$. Therefore part (c) follows.

$(\mathrm{c}) \Rightarrow(\mathrm{d})$. Fix $\theta \in S$. For each $n=0,1, \ldots, m-1$,

$$
x^{n}=\sum_{j=0}^{n} S(n, j) x(x-1) \ldots(x-j+1)
$$

where $S(n, j)(0 \leq j \leq n)$ are Stirling numbers of the second kind, so by (c) we have

$$
\begin{aligned}
& \sum_{\substack{I \subseteq\{1, \ldots, k\} \\
\left\{\Sigma_{s \in I} 1 / \beta_{s}\right\}=\theta}}(-1)^{|I|}\left[\sum_{s \in I} 1 / \beta_{s}\right]^{n} e^{2 \pi i \Sigma_{s \in I} \alpha_{s} / \beta_{s}} \\
& =\sum_{j=0}^{n} j ! S(n, j) \sum_{\substack{I \subseteq\{1, \ldots, k\} \\
\left\{\Sigma_{s \in I} 1 / \beta_{s}\right\}=\theta}}(-1)^{|I|}\left(\begin{array}{c}
{\left[\sum_{s \in I} 1 / \beta_{s}\right]} \\
j
\end{array}\right) e^{2 \pi i \Sigma_{s \in I} \alpha_{s} / \beta_{s}}=0,
\end{aligned}
$$

i.e.

$$
\sum_{v \in V(\theta)} \sum_{\substack{I \subseteq\{1, \ldots, k\} \\ \Sigma_{s} \in I 1 / \beta_{s}=v}}(-1)^{|I|} e^{2 \pi i \Sigma_{s \in I} \alpha_{s} / \beta_{s}}[v]^{n}=0
$$


Cas e 1: $|V(\theta)| \leq m$. In this case

$$
\sum_{v \in V(\theta)}[v]^{n} f(v)=0 \quad \text { for every } n=0,1, \ldots,|V(\theta)|-1 .
$$

Hence (8) holds since $f(v)=0$ for all $v \in V(\theta)$ (Vandermonde).

Case 2: $|V(\theta)|>m$. In this case, $U(\theta) \subset V(\theta)$ and

$$
\sum_{v \in U(\theta)}[v]^{n} f(v)+\sum_{v \in V(\theta) \backslash U(\theta)}[v]^{n} f(v)=0
$$

for each $n=0,1, \ldots, m-1$. According to Lemma 4 ,

$$
\sum_{v \in V(\theta)} a_{u v} f(v)=\sum_{v \in V(\theta)}\left(\prod_{\substack{x \in U(\theta) \\ x \neq u}} \frac{[x]-[v]}{[x]-[u]}\right) f(v)=0 \quad \text { for all } u \in U(\theta) .
$$

So in either case we have (8).

$(\mathrm{d}) \Rightarrow(\mathrm{a})$. Assume that (d) holds. Let $\theta \in S$. For $u, v \in U(\theta)$,

$$
a_{u v}=\prod_{\substack{x \in U(\theta) \\ x \neq u}} \frac{x-v}{x-u}= \begin{cases}1 & \text { if } u=v, \\ 0 & \text { if } u \neq v .\end{cases}
$$

Case 1: $|V(\theta)| \leq m$. In this case $V(\theta) \subseteq U(\theta)$. As

$$
f(u)=\sum_{v \in V(\theta)} a_{u v} f(v)=0 \quad \text { for each } u \in V(\theta),
$$

we get

$$
\sum_{v \in V(\theta)} f(v)[v]^{n}=0 \quad \text { for all } n=0,1,2, \ldots
$$

Case 2: $|V(\theta)|>m$. In this case $U(\theta) \subset V(\theta)$, so for any $u \in U(\theta)$ and $v \in V(\theta)$ we have $\{u\}=\{v\}=\theta$ and hence $[u]-[v]=u-v$. Since

$$
\sum_{v \in V(\theta)}\left(\prod_{\substack{x \in U(\theta) \\ x \neq u}} \frac{[x]-[v]}{[x]-[u]}\right) f(v)=\sum_{v \in V(\theta)} a_{u v} f(v)=0
$$

for every $u \in U(\theta)$, it follows from Lemma 4 that

$$
\sum_{v \in V(\theta)} f(v)[v]^{n}=\sum_{v \in U(\theta)}[v]^{n} f(v)+\sum_{v \in V(\theta) \backslash U(\theta)}[v]^{n} f(v)=0
$$

for all $n=0,1, \ldots, m-1$.

In both cases,

$$
\sum_{v \in V(\theta)} f(v)[v]^{n}=0 \quad \text { for } n=0,1, \ldots, m-1 .
$$


Thus for each nonnegative integer $n<m$,

$$
\begin{aligned}
\sum_{v \in V(\theta)} f(v)\left(\begin{array}{c}
{[v]} \\
n
\end{array}\right) & =\sum_{v \in V(\theta)} f(v) \sum_{j=0}^{n}(-1)^{n-j} s(n, j)[v]^{j} / n ! \\
& =\frac{1}{n !} \sum_{j=0}^{n}(-1)^{n-j} s(n, j) \sum_{v \in V(\theta)} f(v)[v]^{j}=0,
\end{aligned}
$$

where $s(n, j)(0 \leq j \leq n)$ are Stirling numbers of the first kind, i.e.

$$
\begin{aligned}
& \sum_{\substack{I \subseteq\{1, \ldots, k\} \\
\left\{\Sigma_{s \in I} 1 / \beta_{s}\right\}=\theta}}(-1)^{|I|}\left(\begin{array}{c}
{\left[\sum_{s \in I} 1 / \beta_{s}\right]} \\
n
\end{array}\right) e^{2 \pi i \Sigma_{s \in I} \alpha_{s} / \beta_{s}} \\
& =\sum_{v \in V(\theta)} \sum_{\substack{I \subseteq\{1, \ldots, k\} \\
\Sigma_{s \in I} 1 / \beta_{s}=v}}\left(\begin{array}{c}
{[v]} \\
n
\end{array}\right)(-1)^{|I|} e^{2 \pi i \Sigma_{s \in I} \alpha_{s} / \beta_{s}}=0 .
\end{aligned}
$$

Therefore by the proof of $(b) \Rightarrow(c)$,

$$
\begin{aligned}
& \lim _{r \rightarrow 1} \sum_{\substack{I \subseteq\{1, \ldots, k\} \\
\left\{\Sigma_{s \in I} 1 / \beta_{s}\right\}=\theta}}(-1)^{|I|} r^{\Sigma_{s \in I} 1 / \beta_{s}} e^{2 \pi i \Sigma_{s \in I} \alpha_{s} / \beta_{s}} /(1-r)^{m-1} \\
& =\lim _{t \rightarrow 0} \sum_{n=0}^{m-1}(-1)^{n}\left(\sum_{\substack{I \subseteq\{1, \ldots, k\} \\
\left\{\Sigma_{s \in I} 1 / \beta_{s}\right\}=\theta}}(-1)^{|I|}\left(\begin{array}{c}
{\left[\sum_{s \in I} 1 / \beta_{s}\right]} \\
n
\end{array}\right) e^{2 \pi i \Sigma_{s \in I} \alpha_{s} / \beta_{s}}\right) t^{n-m+1} \\
& =0 .
\end{aligned}
$$

Now for every integer $x$,

$$
\begin{aligned}
& \prod_{s=1}^{k}\left(1-r^{1 / \beta_{s}} e^{2 \pi i\left(\alpha_{s}-x\right) / \beta_{s}}\right) \\
& =\sum_{I \subseteq\{1, \ldots, k\}}(-1)^{|I|} r^{\Sigma_{s \in I} 1 / \beta_{s}} e^{2 \pi i \Sigma_{s \in I}\left(\alpha_{s}-x\right) / \beta_{s}} \\
& =\sum_{\theta \in S} e^{-2 \pi i x \theta} \sum_{\substack{I \subseteq\{1, \ldots, k\} \\
\left\{\Sigma_{s \in I} 1 / \beta_{s}\right\}=\theta}}(-1)^{|I|} r^{\Sigma_{s} \in I 1 / \beta_{s}} e^{2 \pi i \Sigma_{s} \in I \alpha_{s} / \beta_{s}} \\
& =\sum_{\theta \in S} e^{-2 \pi i x \theta} o\left((1-r)^{m-1}\right)=o\left((1-r)^{m-1}\right) \quad(r \rightarrow 1) .
\end{aligned}
$$

Applying Lemma 1 we then obtain part (a).

The proof of Theorem 1 is now complete. 
3. Reciprocals of common differences. In $1989 \mathrm{M}$. Z. Zhang [19] showed the following surprising result analytically: Provided that (2) is a cover of $\mathbb{Z}, \sum_{s \in I} 1 / n_{s} \in \mathbb{Z}^{+}$for some $I \subseteq\{1, \ldots, k\}$. Here we give

Theorem 2. Let (1) be a cover of $\mathbb{Z}$. Then for any $J \subseteq\{1, \ldots, k\}$ there is an $I \subseteq\{1, \ldots, k\}$ with $I \neq J$ such that

$$
\sum_{s \in I} \frac{1}{\beta_{s}}-\sum_{s \in J} \frac{1}{\beta_{s}} \in \mathbb{Z}
$$

Proof. Set $\theta=\left\{\sum_{s \in J} 1 / \beta_{s}\right\}$. By Theorem 1,

$$
\sum_{\substack{I \subseteq\{1, \ldots, k\} \\
\left\{\Sigma_{s \in I} 1 / \beta_{s}\right\}=\theta}}(-1)^{|I|}\left(\begin{array}{c}
{\left[\sum_{s \in I} 1 / \beta_{s}\right]} \\
0
\end{array}\right) e^{2 \pi i \Sigma_{s \in I} \alpha_{s} / \beta_{s}}=0,
$$

that is,

$$
\sum_{\substack{J \neq I \subseteq\{1, \ldots, k\} \\\left\{\Sigma_{s \in I} 1 / \beta_{s}\right\}=\theta}}(-1)^{|I|} e^{2 \pi i \Sigma_{s \in I} \alpha_{s} / \beta_{s}}=-(-1)^{|J|} e^{2 \pi i \Sigma_{s \in J} \alpha_{s} / \beta_{s}} .
$$

Therefore

$$
\left\{I \subseteq\{1, \ldots, k\}: I \neq J \text { and }\left\{\sum_{s \in I} \frac{1}{\beta_{s}}\right\}=\theta\right\} \neq \emptyset .
$$

We are done.

In the case $J=\emptyset$, Theorem 2 yields a generalization of Zhang's result ([19]).

Provided that (1) is an $m$-cover of $\mathbb{Z}$ with $m \in \mathbb{Z}^{+}$, Theorem 2 asserts that for any $J \subseteq\{1, \ldots, k\}$,

$$
S(J)=\left\{I \subseteq\{1, \ldots, k\}: I \neq J \text { and } \sum_{s \in I} \frac{1}{\beta_{s}}-\sum_{s \in J} \frac{1}{\beta_{s}} \in \mathbb{Z}\right\}
$$

is nonempty. This becomes trivial if

$$
\sum_{s \in I} \frac{1}{\beta_{s}}=\sum_{s \in J} \frac{1}{\beta_{s}} \quad \text { for some } I \subseteq\{1, \ldots, k\} \text { with } I \neq J .
$$

What can we say about

$$
Z(J)=\left\{\sum_{s \in I} \frac{1}{\beta_{s}}-\sum_{s \in J} \frac{1}{\beta_{s}}: I \in S(J)\right\}
$$

if it does not contain zero? The following theorem gives us more information.

TheOREM 3. Assume that (1) is an $m$-cover of $\mathbb{Z}$. Let $J$ be a subset of $\{1, \ldots, k\}$ such that $(11)$ fails, i.e. $0 \notin Z(J)$ where $S(J)$ and $Z(J)$ are given by (10) and (12). Then 
(i) $|Z(J)| \geq m$ and hence

$$
\sum_{s=1}^{k} \frac{1}{\beta_{s}} \geq m d(J)+\left\{\sum_{s \in J} \frac{1}{\beta_{s}}\right\} \geq m
$$

where $d(J)$ is the least positive integer that can be written as the difference of two (distinct) numbers of the form

$$
\sum_{s \in I} \frac{1}{\beta_{s}} \in \mathbb{Z}+\sum_{s \in J} \frac{1}{\beta_{s}} \quad \text { where } I \subseteq\{1, \ldots, k\} .
$$

(ii) When $d(J) \geq\left[\sum_{s=1}^{k} 1 / \beta_{s}\right] / m, d(J)$ equals $\left[\sum_{s=1}^{k} 1 / \beta_{s}\right] / m$ and $d i$ vides $\left[\sum_{s \in J} 1 / \beta_{s}\right]$, and for every $n=0,1, \ldots, m$ there exist at least

$$
\left(\begin{array}{c}
m \\
n
\end{array}\right) /\left(\begin{array}{c}
m \\
m\left[\sum_{s \in J} 1 / \beta_{s}\right] /\left[\sum_{s=1}^{k} 1 / \beta_{s}\right]
\end{array}\right)
$$

subsets $I$ of $\{1, \ldots, k\}$ such that

$$
\sum_{s \in I} \frac{1}{\beta_{s}}=\frac{n}{m}\left[\sum_{s=1}^{k} \frac{1}{\beta_{s}}\right]+\left\{\sum_{s \in J} \frac{1}{\beta_{s}}\right\}
$$

hence

$$
|S(J)| \geq 2^{m} /\left(\begin{array}{c}
m \\
m\left[\sum_{s \in J} 1 / \beta_{s}\right] /\left[\sum_{s=1}^{k} 1 / \beta_{s}\right]
\end{array}\right)-1 \quad \text { and } \quad|Z(J)|=m .
$$

Proof. Let $\theta=\left\{\sum_{s \in J} 1 / \beta_{s}\right\}, V(\theta), U(\theta)$ and $f(x)$ be as in Theorem 1. If $|V(\theta)| \leq m$, then $V(\theta) \subseteq U(\theta)$, hence by Theorem 1 for all $u \in V(\theta) \subseteq$ $U(\theta)$,

$$
f(u)=\sum_{v \in V(\theta)}\left(\prod_{\substack{x \in U(\theta) \\ x \neq u}} \frac{x-v}{x-u}\right) f(v)=0
$$

which is impossible since $0 \notin Z(J)$ and

$$
f\left(\sum_{s \in J} \frac{1}{\beta_{s}}\right)=(-1)^{|J|} e^{2 \pi i \Sigma_{s \in J} \alpha_{s} / \beta_{s}} \neq 0 .
$$

Thus $|V(\theta)|>m$.

(i) Let $v_{0}<v_{1}<\ldots<v_{m}$ be the first $m+1$ elements of $V(\theta)$ in ascending order. Clearly

$$
1+|Z(J)|=|Z(J) \cup\{0\}|=\left|\left\{v-\sum_{s \in J} \frac{1}{\beta_{s}}: v \in V(\theta)\right\}\right|=|V(\theta)| \geq m+1
$$

and

$$
\sum_{s=1}^{k} \frac{1}{\beta_{s}} \geq \max _{v \in V(\theta)} v \geq v_{m}=\sum_{i=0}^{m-1}\left(v_{i+1}-v_{i}\right)+v_{0} \geq \operatorname{md}(J)+\theta .
$$


(ii) If $|V(\theta)|>m+1$ then

$$
\sum_{s=1}^{k} \frac{1}{\beta_{s}} \geq \max _{v \in V(\theta)} v \geq v_{m}+1 \geq 1+m d(J)+\theta .
$$

Now suppose that $d(J) \geq\left[\sum_{s=1}^{k} 1 / \beta_{s}\right] / m$. Then we must have $|V(\theta)|=$ $m+1$, thus $V(\theta)=\left\{v_{0}, v_{1}, \ldots, v_{m}\right\}$ and $|Z(J)|=|V(\theta)|-1=m$. As

$$
\begin{gathered}
m d(J) \geq\left[\sum_{s=1}^{k} \frac{1}{\beta_{s}}\right] \geq\left[v_{m}\right]=v_{0}-\theta+\sum_{i=0}^{m-1}\left(v_{i+1}-v_{i}\right) \geq\left[v_{0}\right]+m d(J), \\
m d(J)=\left[\sum_{s=1}^{k} \frac{1}{\beta_{s}}\right], \quad\left[v_{0}\right]=0
\end{gathered}
$$

and

$$
\left[v_{n}\right]=v_{0}-\theta+\sum_{i=0}^{n-1}\left(v_{i+1}-v_{i}\right)=0+\sum_{i=0}^{n-1} d(J)=n d(J)
$$

for $n=1, \ldots, m$.

Choose $0 \leq j \leq m$ such that $v_{j}=\sum_{s \in J} 1 / \beta_{s}$. Then

$$
j=\frac{\left[v_{j}\right]}{d(J)}=m\left[\sum_{s \in J} \frac{1}{\beta_{s}}\right] /\left[\sum_{s=1}^{k} \frac{1}{\beta_{s}}\right] .
$$

Set

$$
U^{\prime}(\theta)=\left\{v_{i}: 0 \leq i \leq m, i \neq j\right\} .
$$

By Theorem 1 , for any $n=0,1, \ldots, m$ with $n \neq j$,

$$
\begin{aligned}
0 & =\sum_{v \in V(\theta)}\left(\prod_{\substack{x \in U^{\prime}(\theta) \\
x \neq v_{n}}} \frac{x-v}{x-v_{n}}\right) f(v)=\sum_{t=0}^{m}\left(\prod_{\substack{i=0 \\
i \neq j, n}}^{m} \frac{v_{i}-v_{t}}{v_{i}-v_{n}}\right) f\left(v_{t}\right) \\
& =\sum_{t=0}^{m}\left(\prod_{\substack{i=0 \\
i \neq j, n}}^{m} \frac{i d(J)+\theta-(t d(J)+\theta)}{i d(J)+\theta-(n d(J)+\theta)}\right) f\left(v_{t}\right) \\
& =\sum_{t=0}^{m}\left(\prod_{\substack{i=0 \\
i \neq j, n}}^{m} \frac{i-t}{i-n}\right) f\left(v_{t}\right) \\
& =f\left(v_{n}\right)+\left(\prod_{\substack{i=0 \\
i \neq j, n}}^{m} \frac{i-j}{i-n}\right) f\left(v_{j}\right) .
\end{aligned}
$$


Since

$$
\begin{aligned}
\prod_{\substack{i=0 \\
i \neq j, n}}^{m} \frac{i-j}{i-n} & =\frac{\prod_{i=0, i \neq j}^{m}(i-j)}{n-j} / \frac{\prod_{i=0, i \neq n}^{m}(i-n)}{j-n} \\
& =-\frac{\prod_{i=0}^{j-1}(i-j) \cdot \prod_{i=j+1}^{m}(i-j)}{\prod_{i=0}^{n-1}(i-n) \cdot \prod_{i=n+1}^{m}(i-n)} \\
& =-\frac{(-1)^{j} j !(m-j) !}{(-1)^{n} n !(m-n) !}=(-1)^{j-n+1}\left(\begin{array}{c}
m \\
n
\end{array}\right) /\left(\begin{array}{c}
m \\
j
\end{array}\right),
\end{aligned}
$$

we have

$$
\begin{aligned}
\sum_{\substack{I \subseteq\{1, \ldots, k\} \\
\Sigma_{s \in I} 1 / \beta_{s}=n d(J)+\theta}}(-1)^{|I|} e^{2 \pi i \Sigma_{s \in I} \alpha_{s} / \beta_{s}} \\
\quad=f\left(v_{n}\right)=-(-1)^{j-n+1}\left(\begin{array}{c}
m \\
n
\end{array}\right)\left(\begin{array}{c}
m \\
j
\end{array}\right)^{-1} f\left(\sum_{s \in J} \frac{1}{\beta_{s}}\right) \\
=(-1)^{j-n}\left(\begin{array}{c}
m \\
n
\end{array}\right)\left(\begin{array}{c}
m \\
j
\end{array}\right)^{-1}(-1)^{|J|} e^{2 \pi i \Sigma_{s \in J} \alpha_{s} / \beta_{s}}
\end{aligned}
$$

and hence

$$
\begin{aligned}
\left|\left\{I \subseteq\{1, \ldots, k\}: \sum_{s \in I} \frac{1}{\beta_{s}}=\frac{n}{m}\left[\sum_{s=1}^{k} \frac{1}{\beta_{s}}\right]+\left\{\sum_{s \in J} \frac{1}{\beta_{s}}\right\}\right\}\right| \\
=\sum_{\substack{I \subseteq\{1, \ldots, k\} \\
\Sigma_{s \in I} 1 / \beta_{s}=n d(J)+\theta}} 1 \\
\geq\left|\sum_{\substack{I \subseteq\{1, \ldots, k\} \\
\Sigma_{s \in I} 1 / \beta_{s}=n d(J)+\theta}}(-1)^{|I|} e^{2 \pi i \Sigma_{s \in I} \alpha_{s} / \beta_{s}}\right|=\left(\begin{array}{c}
m \\
n
\end{array}\right) /\left(\begin{array}{c}
m \\
j
\end{array}\right) ;
\end{aligned}
$$

therefore

$$
\begin{aligned}
1+|S(J)| & =\left|\left\{I \subseteq\{1, \ldots, k\}: \sum_{s \in I} \frac{1}{\beta_{s}} \in V(\theta)\right\}\right| \\
& =\sum_{n=0}^{m}\left|\left\{I \subseteq\{1, \ldots, k\}: \sum_{s \in I} \frac{1}{\beta_{s}}=v_{n}=n d(J)+\theta\right\}\right| \\
& \geq \sum_{n=0}^{m}\left(\begin{array}{c}
m \\
n
\end{array}\right) /\left(\begin{array}{c}
m \\
j
\end{array}\right)=2^{m} /\left(\begin{array}{c}
m \\
j
\end{array}\right) .
\end{aligned}
$$

This ends the proof.

Now let us apply Theorem 3 to those $m$-covers (1) with $\sum_{s=1}^{k} 1 / \beta_{s}=m$. 
TheOREM 4. Let (1) be an $m$-cover of $\mathbb{Z}$ with $\sum_{s=1}^{k} 1 / \beta_{s}=m \in \mathbb{Z}^{+}$, which happens if (1) is an exact $m$-cover of $\mathbb{Z}$ by residue classes. Then

(i) For every $l=1, \ldots, k-1$ we have

$$
\sum_{s=l+1}^{k} \frac{1}{\beta_{s}} \geq \frac{1}{\max \left\{\beta_{1}, \ldots, \beta_{l}\right\}} .
$$

(ii) For any $\emptyset \neq J \subset\{1, \ldots, k\}$ there exists an $I \subseteq\{1, \ldots, k\}$ with $I \neq J$ such that

$$
\sum_{s \in I} \frac{1}{\beta_{s}}=\sum_{s \in J} \frac{1}{\beta_{s}}
$$

furthermore when $\sum_{s \in J} 1 / \beta_{s} \in \mathbb{Z}$ there are at least

$$
\left(\begin{array}{c}
m \\
\sum_{s \in J} 1 / \beta_{s}
\end{array}\right) \geq m>1
$$

subsets $I$ of $\{1, \ldots, k\}$ satisfying (16).

Proof. (i) For $l=1, \ldots, k-1$ (15) follows from part (ii) in the case $J=\{l+1, \ldots, k\}$, so we proceed to the proof of part (ii).

(ii) If (11) fails then by part (i) of Theorem 3 and the equality $\sum_{s=1}^{k} 1 / \beta_{s}$ $=m$ we must have

$$
\left\{\sum_{s \in J} \frac{1}{\beta_{s}}\right\}=0, \quad \text { i.e. } \quad \sum_{s \in J} \frac{1}{\beta_{s}} \in \mathbb{Z} .
$$

Observe that

$$
0<\sum_{s \in J} \frac{1}{\beta_{s}}<\sum_{s=1}^{k} \frac{1}{\beta_{s}}=m .
$$

If $\sum_{s \in J} 1 / \beta_{s} \in \mathbb{Z}$, then $m>1$ and $\sum_{s \in J} 1 / \beta_{s}=n$ for some $n=1, \ldots, m-1$, by part (ii) of Theorem 3 there are at least $\left(\begin{array}{c}m \\ n\end{array}\right) /\left(\begin{array}{c}m \\ m\end{array}\right)=\left(\begin{array}{c}m \\ n\end{array}\right) \geq m$ subsets $I$ of $\{1, \ldots, k\}$ such that

$$
\sum_{s \in I} \frac{1}{\beta_{s}}=\frac{n}{m}\left[\sum_{s=1}^{k} \frac{1}{\beta_{s}}\right]+\left\{\sum_{1 \leq s \leq k} \frac{1}{\beta_{s}}\right\}=n=\sum_{s \in J} \frac{1}{\beta_{s}} .
$$

We are done.

Remark. In 1992 Z. W. Sun ([17]) proved that if (2) is an exact $m$-cover of $\mathbb{Z}$ then for each $n=1, \ldots, m$ there exist at least $\left(\begin{array}{c}m \\ n\end{array}\right)$ subsets $I$ of $\{1, \ldots, k\}$ such that $\sum_{s \in I} 1 / n_{s}$ equals $n$. The lower bounds $\left(\begin{array}{c}m \\ n\end{array}\right)(1 \leq n \leq m)$ are best possible, and the Riemann zeta function was used in the proof.

From Theorem 3 we can also deduce the following theorem which extends Zhang's result ([19]) and the theorem of Sun [17] even in the case $l=k$. 
TheOREM 5. Let (1) be an $m$-cover of $\mathbb{Z}$ and $l$ a positive integer not exceeding $k$ such that

$$
\min \left\{1, \frac{1}{\beta_{1}}, \ldots, \frac{1}{\beta_{l}}\right\}>\sum_{l<t \leq k} \frac{1}{\beta_{t}},
$$

where $\sum_{l<t \leq k} 1 / \beta_{t}$ is considered to be zero for $l=k$. Then

(i) There are at least $m$ positive integers representable by

$$
\sum_{s \in I} \frac{1}{\beta_{s}}-\sum_{l<t \leq k} \frac{1}{\beta_{t}}, \quad \text { where } I \subseteq\{1, \ldots, k\},
$$

thus we have

$$
\sum_{s=1}^{l} \frac{1}{\beta_{s}}=\sum_{s=1}^{k} \frac{1}{\beta_{s}}-\sum_{l<t \leq k} \frac{1}{\beta_{t}} \geq m .
$$

(ii) Provided that any positive integer less than $\left[\sum_{s=1}^{k} 1 / \beta_{s}\right] / m$ cannot be expressed as the difference of two integers of the form (18), $\left[\sum_{s=1}^{k} 1 / \beta_{s}\right]$ is divisible by $m$ and for each $n=0,1, \ldots, m$ there are at least $\left(\begin{array}{c}m \\ n\end{array}\right)$ subsets I of $\{1, \ldots, k\}$ such that

$$
\sum_{s \in I} \frac{1}{\beta_{s}}=\frac{n}{m}\left[\sum_{s=1}^{k} \frac{1}{\beta_{s}}\right]+\sum_{l<t \leq k} \frac{1}{\beta_{t}},
$$

hence there exist at least $2^{m}-1$ subsets $I$ of $\{1, \ldots, k\}$ with

$$
\sum_{s \in I} \frac{1}{\beta_{s}} \in \mathbb{Z}^{+}+\sum_{l<t \leq k} \frac{1}{\beta_{t}} .
$$

Proof. Let $J=\{1 \leq t \leq k: t>l\}$. By (17),

$$
\left[\sum_{t \in J} \frac{1}{\beta_{t}}\right]=0 \quad \text { and } \quad\left\{\sum_{t \in J} \frac{1}{\beta_{t}}\right\}=\sum_{l<t \leq k} \frac{1}{\beta_{t}} .
$$

For any $I \subseteq\{1, \ldots, k\}$, if $I \subset J$ then

$$
0<\sum_{t \in J} \frac{1}{\beta_{t}}-\sum_{s \in I} \frac{1}{\beta_{s}}<1,
$$

and if $I \nsubseteq J$ then

$$
\sum_{s \in I} \frac{1}{\beta_{s}}-\sum_{t \in J} \frac{1}{\beta_{t}} \geq \min \left\{\frac{1}{\beta_{s}}: 1 \leq s \leq l\right\}-\sum_{l<t \leq k} \frac{1}{\beta_{t}}>0 .
$$

So (11) fails, moreover $Z(J)$ given by (12) contains only positive integers. Applying Theorem 3 we obtain the desired results. 
Erdös conjectured (before 1950) that if (2) is a cover of $\mathbb{Z}$ with $1<n_{1}<$ $n_{2}<\ldots<n_{k}$ then $\sum_{s=1}^{k} 1 / n_{s}>1$. H. Davenport, L. Mirsky, D. Newman and $\mathrm{R}$. Radó confirmed this conjecture (independently) by proving that if (2) is an exact cover of $\mathbb{Z}$ with $1<n_{1} \leq \ldots \leq n_{k-1} \leq n_{k}$ then $n_{k-1}=n_{k}$. For further improvements see Znám [20], M. Newman [10], Porubský [11, 12], M. A. Berger, A. Felzenbaum and A. S. Fraenkel [1]. The best record in this direction is the following result due to the author which is partially announced in [15] and completely proved in [16]: Let $\lambda_{1}, \ldots, \lambda_{k}$ be complex numbers and $n_{0} \in \mathbb{Z}^{+}$a period of the function

$$
\sigma(x)=\sum_{\substack{s=1 \\ x \equiv a_{s}\left(\bmod n_{s}\right)}}^{k} \lambda_{s} .
$$

If $d \in \mathbb{Z}^{+}$does not divide $n_{0}$ and

$$
\sum_{\substack{s=1 \\ d \mid n_{s}, a_{s} \equiv a(\bmod d)}}^{k} \frac{\lambda_{s}}{n_{s}} \neq 0 \quad \text { for some integer } a
$$

then

$$
\left|\left\{a_{s} \bmod d: 1 \leq s \leq k, d \mid n_{s}\right\}\right| \geq \min _{\substack{0 \leq s \leq k \\ d \nmid n_{s}}} \frac{d}{\operatorname{gcd}\left(d, n_{s}\right)} \geq p(d)
$$

where $p(d)$ is the least prime divisor of $d$. Here we have

TheOREM 6. Let (1) be an m-cover of $\mathbb{Z}$ with $\beta_{1} \leq \ldots \leq \beta_{k-l}<$ $\beta_{k-l+1}=\ldots=\beta_{k}$ where $1 \leq l<k$. Then either

$$
l \geq \beta_{k} / \max \left\{1, \beta_{k-l}\right\} \quad\left(>1 \text { if } \beta_{k}>1\right),
$$

or there are at least $m$ positive integers in the form

$$
\sum_{s \in I} \frac{1}{\beta_{s}}-\frac{l}{\beta_{k}}, \quad \text { where } I \subseteq\{1, \ldots, k\},
$$

and hence

$$
\sum_{s=1}^{k} \frac{1}{\beta_{s}}>\sum_{s=1}^{k-l} \frac{1}{\beta_{s}}=\sum_{s=1}^{k} \frac{1}{\beta_{s}}-\frac{l}{\beta_{k}} \geq m .
$$

(Also, $\sum_{s=1}^{k} 1 / \beta_{s}>\sum_{s=1}^{k} 1 / \beta_{k} \geq k \geq m$ if $\beta_{k} \leq 1$.)

Proof. Clearly $l<\beta_{k} / \max \left\{1, \beta_{k-l}\right\}$ if and only if

$$
\min \left\{1, \frac{1}{\beta_{1}}, \ldots, \frac{1}{\beta_{k-l}}\right\}>\sum_{k-l<t \leq k} \frac{1}{\beta_{t}}\left(=l / \beta_{k}\right) .
$$

Therefore Theorem 6 follows from part (i) of Theorem 5 . 
Note that when $\beta_{k-l} \geq 1$ and $\beta_{k} / \beta_{k-l} \in \mathbb{Z}$

$$
\beta_{k} / \max \left\{1, \beta_{k-l}\right\}=\beta_{k} / \beta_{k-l} \geq p\left(\beta_{k} / \beta_{k-l}\right) \quad\left(\geq p\left(\beta_{k}\right) \text { if } \beta_{k-l}, \beta_{k} \in \mathbb{Z}\right) .
$$

4. Some local-global results. In 1958 S. K. Stein [14] conjectured that whenever the residue classes in (2) are pairwise disjoint and the moduli $n_{1}, \ldots, n_{k}>1$ are distinct there exists an integer $x$ with $1 \leq x \leq 2^{k}$ such that $x$ is not covered by (2). Erdös [6] confirmed this conjecture with $k \cdot 2^{k}$ instead of $2^{k}$. Since the Davenport-Mirsky-Newman-Radó result indicates that an exact cover of $\mathbb{Z}$ by (finitely many) residue classes cannot have its moduli distinct and greater than one, Erdős proposed the stronger conjecture that any system of $k$ residue classes not covering all the integers omits a positive integer not exceeding $2^{k}$. Both conjectures have some localglobal character. In 1969 R. B. Crittenden and C. L. Vanden Eynden [2] claimed their positive answer to the stronger conjecture. Later in [3] a long indirect and awkward proof was given for $k \geq 20$, the authors concluded the paper with the statements: "Of course it remains to show the conjecture is true for $k<20$. This may be checked by more special arguments."

In 1970 Crittenden and Vanden Eynden [4] conjectured further that if all the moduli $n_{s}$ in (2) are greater than an integer $0 \leq l<k$ then (2) is a cover of $\mathbb{Z}$ if it covers all the integers in the interval $\left[1,2^{k-l}(l+1)\right]$. In contrast with the Crittenden-Vanden Eynden conjecture we give

TheOREM 7. For any $m \in \mathbb{Z}^{+}$, (1) is an $m$-cover of $\mathbb{Z}$ if it covers $2^{k-M}(M+1)$ consecutive integers at least $m$ times, where

$$
M=\max _{1 \leq t \leq k}\left|\left\{1 \leq s \leq k: \beta_{s}=\beta_{t}\right\}\right| .
$$

Proof. Let $\beta>0$ be a number such that $J=\left\{1 \leq s \leq k: \beta_{s}=\beta\right\}$ has cardinality $M$. As

$$
\begin{aligned}
\mid\left\{\left\{\sum_{s \in I} \frac{1}{\beta_{s}}\right\}:\right. & I \subseteq\{1, \ldots, k\}\} \mid \\
& \leq\left|\left\{\sum_{s \in I \cap J} \frac{1}{\beta_{s}}+\sum_{s \in I \backslash J} \frac{1}{\beta_{s}}: I \subseteq\{1, \ldots, k\}\right\}\right| \\
& \leq\left|\left\{\sum_{s \in I} \frac{1}{\beta}: I \subseteq J\right\}\right| \cdot\left|\left\{\sum_{s \in I} \frac{1}{\beta_{s}}: I \subseteq\{1, \ldots, k\} \backslash J\right\}\right| \\
& \leq\left|\left\{\frac{|I|}{\beta}: I \subseteq J\right\}\right| \cdot|\{I: I \subseteq\{1, \ldots, k\} \backslash J\}| \\
& =(|J|+1) \cdot 2^{k-|J|}=2^{k-M}(M+1),
\end{aligned}
$$

Theorem 1 implies Theorem 7 . 
The following example noted by Crittenden and Vanden Eynden [4] shows that the number $g(k, M)=2^{k-M}(M+1)$ in Theorem 7 is best possible.

Example. Let $M=n-1 \in \mathbb{Z}^{+}$. Consider the system $A$ consisting of the following $k \geq M$ residue classes:

$$
\begin{gathered}
1+n \mathbb{Z}, \quad 2+n \mathbb{Z}, \quad \ldots, \quad M+n \mathbb{Z}, \\
n+2 n \mathbb{Z}, \quad 2 n+2^{2} n \mathbb{Z}, \quad \ldots, \quad 2^{k-M-1} n+2^{k-M} n \mathbb{Z} .
\end{gathered}
$$

Observe that $A$ together with $2^{k-M} n \mathbb{Z}$ forms an exact cover of $\mathbb{Z}$. So $A$ covers positive integers from 1 to $2^{k-M}(M+1)-1$, but it does not cover all the integers.

Result (II) stated in Section 1 follows from Theorem 7 and Example, since (1) covers $\alpha+\beta x$ (where $\alpha \in \mathbb{R}, \beta \in \mathbb{R}^{+}$and $x \in \mathbb{Z}$ ) at least $m$ times if and only if $\left\{\frac{\alpha_{s}-\alpha}{\beta}+\frac{\beta_{s}}{\beta} \mathbb{Z}\right\}_{s=1}^{k}$ covers $x$ at least $m$ times, and $2^{k-l}(l+1) \geq$ $2^{k-M}(M+1)$ if $k \geq M \geq l>0$. (The case $l=0$ can be reduced to the case $l=1$.)

5. Several open problems. Theorem 1 tells us that (2) is a cover of $\mathbb{Z}$ if it covers integers from 1 to

$$
\left|\left\{\left\{\sum_{s \in I} \frac{1}{\beta_{s}}\right\}: I \subseteq\{1, \ldots, k\}\right\}\right| \leq 2^{k} \leq 2^{n_{1}+\ldots+n_{k}} .
$$

This suggests

Problem 1. Can we find a polynomial $P$ with integer coefficients such that a finite system (2) of residue classes forms a cover of $\mathbb{Z}$ whenever it covers all positive integers not exceeding $P\left(n_{1}+\ldots+n_{k}\right)$ ?

In 1973 L. J. Stockmeyer and A. R. Meyer proved that the problem whether there exists an integer not covered by a given (2) is NP-complete. In 1991 S. P. Tung [18] extended this result to algebraic integer rings. If the required $P$ in Problem 1 exists, then there is a polynomial time algorithm to decide whether $(2)$ covers all the integers or not. So a positive answer to Problem 1 would imply that NP $=\mathrm{P}$.

By appearances Theorems 2-7 involve no roots of unity. Perhaps vast generalizations of them could be made.

Problem 2. Let $A_{1}, \ldots, A_{k}$ be sets of natural numbers having positive densities $d\left(A_{1}\right), \ldots, d\left(A_{k}\right)$ respectively. If no $A_{s}$ contains $m_{s} \in \mathbb{Z}^{+}$ consecutive integers, does $\bigcup_{s=1}^{k} A_{s}$ have density 1 when it covers $m_{1} \ldots m_{k}$ arbitrarily large consecutive integers? Suppose that $\left\{A_{s}\right\}_{s=1}^{k}$ covers all the natural numbers; does there exist, for any $J \subseteq\{1, \ldots, k\}$, an $I \subseteq\{1, \ldots, k\}$ 
with $I \neq J$ such that

$$
\sum_{s \in I} d\left(A_{s}\right)-\sum_{s \in J} d\left(A_{s}\right) \in \mathbb{Z} ?
$$

Problem 3. Let $K$ be an algebraic number field and $O_{K}$ the ring of algebraic integers in $K$. Let $a_{1}, \ldots, a_{k} \in O_{K}$ and $A_{1}, \ldots, A_{k}$ be ideals of $O_{K}$ with norms $N\left(A_{1}\right), \ldots, N\left(A_{k}\right)$ respectively. If $\left\{a_{s}+A_{s}\right\}_{s=1}^{k}$ forms an exact $m$-cover of $O_{K}$ for some $m \in \mathbb{Z}^{+}$, is it true that for any $\emptyset \neq J \subseteq\{1, \ldots, k\}$ there exists a subset $I$ of $\{1, \ldots, k\}$ with $I \neq J$ such that

$$
\sum_{s \in I} \frac{1}{N\left(A_{s}\right)}=\sum_{s \in J} \frac{1}{N\left(A_{s}\right)} ?
$$

Acknowledgements. The author is indebted to the referee for his helpful suggestions.

\section{References}

[1] M. A. Berger, A. Felzenbaum and A. S. Fraenkel, Improvements to the Newman-Znám result for disjoint covering systems, Acta Arith. 50 (1988), 1-13.

[2] R. B. Crittenden and C. L. Vanden Eynden, A proof of a conjecture of Erdös, Bull. Amer. Math. Soc. 75 (1969), 1326-1329.

[3] -, - Any $n$ arithmetic progressions covering the first $2^{n}$ integers cover all integers, Proc. Amer. Math. Soc. 24 (1970), 475-481.

[4] - - - The union of arithmetic progressions with differences not less than $k$, Amer Math. Monthly 79 (1972), 630.

[5] P. Erdös, On integers of the form $2^{k}+p$ and some related problems, Summa Brasil. Math. 2 (1950), 113-123.

[6] - Remarks on number theory IV: Extremal problems in number theory I, Mat. Lapok 13 (1962), 228-255.

[7] - Problems and results on combinatorial number theory III, in: Number Theory Day, M. B. Nathanson (ed.), Lecture Notes in Math. 626, Springer, New York, 1977, 43-72.

[8] - Problems and results in number theory, in: Recent Progress in Analytic Number Theory, H. Halberstam and C. Hooley (eds.), Vol. 1, Academic Press, London, 1981, $1-14$.

[9] R. K. Guy, Unsolved Problems in Number Theory, Springer, New York, 1981.

[10] M. Newman, Roots of unity and covering sets, Math. Ann. 191 (1971), 279-282.

[11] Š. Porubský, Covering systems and generating functions, Acta Arith. 26 (1974/75), 223-231.

[12] —, On m times covering systems of congruences, ibid. 29 (1976), 159-169.

[13] - Results and problems on covering systems of residue classes, Mitt. Math. Sem. Giessen 1981, no. 150, 1-85.

[14] S. K. Stein, Unions of arithmetic sequences, Math. Ann. 134 (1958), 289-294.

[15] Z. W. Sun, Several results on systems of residue classes, Adv. in Math. (Beijing) 18 (1989), 251-252.

[16] -, An improvement to the Znám-Newman result, Chinese Quart. J. Math. 6 (3) (1991), 90-96. 
[17] Z. W. Sun, On exactly $m$ times covers, Israel. J. Math. 77 (1992), 345-348.

[18] S. P. Tung, Complexity of sentences over number rings, SIAM J. Comp. 20 (1991), $126-143$

[19] M. Z. Zhang, A note on covering systems of residue classes, J. Sichuan Univ. (Nat. Sci. Ed.) 26 (1989), Special Issue, 185-188.

[20] Š. Znám, On exactly covering systems of arithmetic sequences, Math. Ann. 180 (1969), 227-232.

[21] - A survey of covering systems of congruences, Acta Math. Univ. Comenian. 40/41 (1982), 59-79.

DEPARTMENT OF MATHEMATICS

NANJING UNIVERSITY

NANJING 210093

PEOPLE'S REPUBLIC OF CHINA

Received on 18.11.1993

and in revised form on 23.8.1994 